\title{
Research on Dynamic Tracking of Seamless Positioning Based on Global Positioning System, Beidou, and Digital Television Multimedia Broadcasting
}

\author{
Cheng-Biao Fu, ${ }^{1,2}$ Shu Gan, ${ }^{1 *}$ Xi-Ping Yuan, ${ }^{1}$ and An-Hong Tian ${ }^{1,2}$ \\ ${ }^{1}$ Faculty of Land Resource Engineering, Kunming University of Science and Technology, \\ Kunming, Yunnan 650093, China \\ ${ }^{2}$ College of Information Engineering, Qujing Normal University, Qujing, Yunnan 655011, China
}

(Received March 28, 2018; accepted August 24, 2018)

Keywords: integrated navigation, seamless positioning, PF algorithm, EKF algorithm

Because of the location failure of the global positioning system (GPS) in dense urban areas and indoor environments, a seamless positioning system based on GPS, Beidou, and digital television multimedia broadcasting (DTMB), which has nonlinear model characteristics, has been proposed. This paper focuses on the dynamic positioning tracking effect of two kinds of nonlinear filtering algorithm under the three-dimensional planar variable turn model (PVTM); that is, the nonlinear filtering algorithm mainly contains an extended Kalman filter (EKF) and a particle filter (PF). Our simulation has been carried out from two aspects; on one hand, the number of particles affects the position tracking precision in the PF algorithm. On the other hand, the location tracking effects of the PF and EKF algorithms have been comparatively analyzed using the same simulation model. Theoretical analysis and simulation results show that the filtering trajectories of EKF and PF are basically consistent with the real trajectory, but the tracking performance of the PF algorithm is higher than that of the EKF algorithm. Error means of 500 and 100 particles are 2.8736 and $5.3601 \mathrm{~m}$, respectively. The error mean of EKF is $11.2065 \mathrm{~m}$. The seamless positioning system can meet the positioning accuracy, coverage, and real-time performance requirements. The feasibility and superiority of the seamless positioning system have been proved. Such studies have positive practical and profound historically significant effects on the positioning system in the development of China's independent intellectual property rights based on DTMB and Beidou signals.

\section{Introduction}

With the rapid development of information technology, demand for positioning accuracy increases under various scenarios, the global positioning system (GPS) has been widely used in unobstructed outdoor environments. However, in dense urban areas and indoor environments, owing to the insufficient number of visible satellites, the obvious phenomenon that GPS cannot achieve accurate positioning is observed. Moreover, the daily activity areas of people are mainly in urban areas and indoor environments, which have various positioning signals. To

*Corresponding author: e-mail: sgbf@kmust.edu.cn

https://doi.org/10.18494/SAM.2018.2067 
ensure the automatic switching and seamless connection of different positioning technologies in various scenarios, seamless navigation and positioning techniques have been developed.

There are currently four digital television (TV) signal standards in the world, namely, digital video broadcasting-terrestrial (DVB-T) in Europe, advanced television systems committee (ATSC) in the United States, integrated service digital broadcast-terrestrial (ISDB-T) in Japan, and digital television multimedia broadcasting (DTMB) in China. DTMB standards were formulated on August 18, 2006 in China with independent intellectual property rights. The DTMB system will fully realize the reception of terrestrial TV in 2020. Compared with the satellite signal, the strength of the DTMB signal is more than $30 \mathrm{~dB}$ and can easily penetrate urban and indoor environments, and the TV signal has a high transmission power, wide coverage, low frequency, high positioning accuracy, and high bandwidth, making it more suitable for precise localization in cities and indoor environments. ${ }^{(1-3)}$ Thus, DTMB signals become an ideal choice for auxiliary satellites in urban areas and indoors. The positioning technology based on TV terrestrial broadcasting signals has a certain research potential outside China. For instance, Rabinowitz and Spilker of Stanford University earlier applied the ATSC standard to a position test, ${ }^{(4)}$ and their results showed that the positioning error was between 3.2 and $23.3 \mathrm{~m}$. In Europe, scholars have begun to study the location service based on the DVB-T signal. Compared with DVB-T, the DTMB standard in China was promulgated later, TV stations perform standard migration and tests, and the research based on a TV terrestrial broadcasting signal is still in its infancy. Le-nan Wu's group in Southeast University made some achievements with digital broadcast television signal radio integrated positioning. ${ }^{(5)}$ In recent years, Wang et al. have engaged in digital TV positioning technology research and proposed a joint positioning method of time frequency in two dimensions, which was based on the pseudo-random noise signal sequence in the header and transmission parameter signaling in the frame body. ${ }^{(6)}$

At present, China is vigorously developing the Beidou satellite navigation system, which is a satellite positioning and navigation system independently developed by China with independent intellectual property rights. Since December 27, 2012, it has officially been providing precise positioning navigation services and two-way messages to most parts of Asia Pacific. Communication services are expected to be fully realized in 2020 to achieve global coverage. However, since the Beidou signal is launched by satellites at high altitudes, the signal is perpendicular to the ground, the receiving power is low, and the Beidou signal is the same as the GPS signal. There is also the problem that positioning cannot be provided in densely populated urban areas and indoors.

To truly achieve seamless positioning and make up for the insufficiency of a satellite navigation system in densely populated urban areas and indoors, a seamless positioning scheme based on GPS, Beidou, and DTMB is proposed in this paper to provide accurate positioning for urban areas and indoor environments. Both DTMB and Beidou are independently developed by China. Combination positioning will become a novel independent positioning technology in China, reduce reliance on GPS positioning technology, and ensure information security while providing seamless positioning. Moreover, the integrated positioning system can be used as an auxiliary or backup for special cases of military navigation and positioning systems. 


\section{Materials and Methods}

\subsection{Seamless positioning}

In order to realize the seamless positioning technology indoors and outdoors, the standards used to evaluate different location areas are based on the position information of the mobile terminal and the number of GPS satellites received. In this paper, a seamless positioning mechanism is designed in three models. Firstly, the GPS- and Beidou-based integrated positioning mode has been used in outdoor areas when the quality of GPS signals is good; that is, if the number of satellites is more than four, we can adopt the GPS- and Beidou-based integrated navigation system to locate signals. Secondly, the DTMB positioning mode has been used in indoor areas; thus, we can fully adopt DTMB to locate GPS signals that are inexistent. Thirdly, the integrated positioning mode based on GPS, Beidou, and DTMB has been used in urban areas when the quality of GPS signals is poor; that is, when the number of satellites is less than four, combination positioning is adopted and the final positioning information is obtained by the fusion of nonlinear filtering algorithms. The concrete realization scheme of seamless positioning technology is shown in Table 1.

In the outdoor environment, the pseudo-range positioning method has been adopted in the integrated positioning system based on GPS and Beidou. There are two kinds of different positioning system models that exist in the integrated positioning system, with each positioning system model having its own clock calculation. Thus, at least five unknown parameters, namely, three-dimensional position coordinates and clock deviation of two systems, are observed. The integrated positioning equation can be written as

$$
\rho_{i}=\sqrt{\left(x_{i}-x_{\mathrm{w}}\right)^{2}+\left(y_{i}-y_{w}\right)^{2}+\left(z_{i}-z_{w}\right)^{2}}+v_{1} a_{1}+v_{2} a_{2} \text {. }
$$

In view of the urban environment, the pseudo-range positioning method has also been adopted in the integrated positioning system based on GPS, Beidou, and DTMB. There are three different positioning system models that exist in the integrated positioning system, with each positioning system model having its own clock calculation. Thus, at least six unknown parameters, namely, three-dimensional position coordinates and clock deviation of three systems, are observed. The integrated positioning equation can be defined as

Table 1

Scheme of seamless positioning.

\begin{tabular}{lcc}
\hline Number of GPS satellites & Location area & Positioning scheme \\
\hline More than four & Outdoor area & Combination positioning of GPS and Beidou \\
Between zero and four & Urban area & Combination positioning of GPS, Beidou, and DTMB \\
Less than zero & Indoor area & DTMB positioning \\
\hline
\end{tabular}




$$
\left\{\begin{array}{c}
\rho_{1}=\sqrt{\left(x_{1}-x_{w}\right)^{2}+\left(y_{1}-y_{w}\right)^{2}+\left(z_{1}-z_{w}\right)^{2}}+v_{1} a_{1}+v_{2} a_{2}+v_{3} a_{3}, \\
\rho_{2}=\sqrt{\left(x_{2}-x_{w}\right)^{2}+\left(y_{2}-y_{w}\right)^{2}+\left(z_{2}-z_{w}\right)^{2}}+v_{1} a_{1}+v_{2} a_{2}+v_{3} a_{3}, \\
\rho_{3}=\sqrt{\left(x_{3}-x_{w}\right)^{2}+\left(y_{3}-y_{w}\right)^{2}+\left(z_{3}-z_{w}\right)^{2}}+v_{1} a_{1}+v_{2} a_{2}+v_{3} a_{3}, \\
\cdots \\
\rho_{n}=\sqrt{\left(x_{n}-x_{w}\right)^{2}+\left(y_{n}-y_{w}\right)^{2}+\left(z_{n}-z_{w}\right)^{2}}+v_{1} a_{1}+v_{2} a_{2}+v_{3} a_{3} .
\end{array}\right.
$$

For the indoor environment, only the DTMB positioning system exists; thus, there are three positioning unknowns, namely, three-dimensional position coordinates. The positioning equation based on the DTMB signal is expressed as

$$
\rho_{i}=\sqrt{\left(x_{i}-x_{\mathrm{w}}\right)^{2}+\left(y_{i}-y_{w}\right)^{2}+\left(z_{i}-z_{w}\right)^{2}}+a_{3},
$$

where $\rho_{i}$ has a pseudo-range value, $\left(x_{i}, y_{i}, z_{i}\right)$ are the coordinates of the GPS or Beidou satellite or the DTMB television tower, $\left(x_{w}, y_{w}, z_{w}\right)$ are the coordinates of the receiver, $a_{1}$ is the clock deviation of the GPS satellite, $a_{1}=c \cdot \Delta t_{G P S}, a_{2}$ is the clock deviation of the Beidou satellite, $a_{2}=c \cdot \Delta t_{\text {Beidou }}, a_{3}$ is the clock deviation of the DTMB signal, and $a_{3}=c \cdot \Delta t_{D T M B}$.

In Eq. (1), when the GPS satellite signal is present, $v_{1}=1$ and $v_{2}=0$, whereas, when the satellite signal is Beidou, $v_{1}=0$ and $v_{2}=1$. In Eq. (2), when the GPS satellite signal is present, $v_{1}=1, v_{2}=0$, and $v_{3}=0$, whereas, when the Beidou satellite signal is present, $v_{1}=0, v_{2}=1$, and $v_{3}=0$. Moreover, when the DTMB satellite signal is present, $v_{1}=0, v_{2}=0$, and $v_{3}=1$.

\subsection{Application of extended Kalman filter (EKF) algorithm in seamless positioning}

The assumed state equation and observation equation model of the nonlinear system can be written as

$$
\left\{\begin{array}{c}
X(k)=f(X(k-1))+W(k-1), \\
Y(k)=h(X(k))+V(k),
\end{array}\right.
$$

where the state and observation equations of noise are separately written as $W(k)$ and $V(k)$, respectively, which are unrelated white-noise sequences. Their covariance matrices are, respectively, $Q(k)$ and $R(k)$.

The EKF algorithm ${ }^{(7-9)}$ should yield the optimal linear estimate $\hat{X}(k \mid j)$ of state $X(k)$ by the observations of $\{Y(1), Y(2), \ldots, Y(j)\}$ at the moment of $j$. The linearization of nonlinear Eq. (4) can be defined as 


$$
\begin{gathered}
X(k) \approx f(\hat{X}(k-1))+\left.\frac{\partial f}{\partial X}\right|_{X(k-1)=\hat{X}(k-1)}[X(k-1)-\hat{X}(k-1)]+W(k-1) \\
=A X(k-1)+W(k-1), \\
Y(k) \approx h(\hat{X}(k))+\left.\frac{\partial h}{\partial X}\right|_{X(k)=\hat{X}(k)}[X(k)-\hat{X}(k)]+V(k) \\
=H X(k)+V(k) .
\end{gathered}
$$

The estimation error is expressed as

$$
\tilde{X}(k \mid j)=X(k)-\hat{X}(k \mid j) .
$$

The state error covariance matrix is written as

$$
P(k \mid j)=E\left[\tilde{X}(k \mid j) \tilde{X}^{\mathrm{T}}(k \mid j)\right] .
$$

The optimal estimate of the EKF algorithm is confirmed when $P(k \mid j)$ is minimum. If $k=j$, which is a filtering problem, $\hat{X}(k), \tilde{X}(k)$, and $P(k)$ are used to denote $\hat{X}(k \mid j), \tilde{X}(k \mid j)$, and $P(k \mid j)$, respectively. The step state prediction is $\hat{X}(k \mid j-1)$ and the error matrix of step state prediction is $P(k \mid k-1)$. The recursive process of state estimation can be shown as follows.

The state estimation update equation is defined as

$$
\hat{X}(k)=A \hat{X}(k-1)+K(k)[Y(k)-H A \hat{X}(k-1)] .
$$

The filter gain equation is described as

$$
\begin{gathered}
K(k)=P(k \mid k-1) H^{\mathrm{T}}\left[H P(k \mid k-1) H^{\mathrm{T}}+R(k)\right]^{-1}, \\
P(k \mid k-1)=A P(k-1) A^{\mathrm{T}}+Q(k-1) .
\end{gathered}
$$

The filtering covariance update equation is written as

$$
P(k)=P(k \mid k-1)-K(k) H P(k \mid k-1) .
$$

If the initial state vector is $X(0)$, the initial state estimation error covariance matrix is $P(0)$, and the EKF algorithm can yield the optimal linear estimate $\hat{X}(k \mid j)$ of state $X(k)$ in accordance with Eqs. (9)-(12) to complete the state estimation of the flight target. 


\subsection{Application of particle filter (PF) algorithm in seamless positioning}

The state and observation equations of the nonlinear system of the stochastic state space model can be shown as

$$
\left\{\begin{array}{l}
x_{k}=f\left(x_{k-1}\right)+u_{k-1} \\
y_{k}=h\left(x_{k}\right)+v_{k}
\end{array}\right.
$$

where $x_{k}$ and $y_{k}$ are the system state and observation vectors at the moment of $K$, and $u_{k}$ and $v_{k}$ are the state process noise and measurement noise, respectively.

The standard PF algorithm ${ }^{(10-12)}$ is composed of sequential importance sampling and resampling, and its specific steps can be shown as follows.

Step 1. Initialization particles and weights. Generate $N \times l$ random sampling points based on the distribution function of the system noise, where $l$ is the dimension of state vector $x_{k}$, $N$ is the number of particles, and $1 / N$ is the initialization weight. Particles and weights can be expressed as $p\left(x_{0}\right)=\left\{x_{0}^{i}, \omega_{0}^{i}\right\}_{i=1}^{N}$.

Step 2. Time update. The next time a particle is predicted as $\hat{x}_{k}^{i}$ from the equation of state.

Step 3. Measurement update. The observation vector is predicted as $\hat{y}_{k}$ by substituting $\hat{x}_{k}{ }_{k}$ into the observation equation.

Step 4. Weight update. $\omega_{k}^{i} \propto \omega_{k-1}^{i} \frac{p\left(y_{k} \mid x_{k}^{i}\right) p\left(x_{k}^{i} \mid x_{k-1}^{i}\right)}{q\left(x_{k}^{i} \mid x_{k-1}^{i}, y_{1: k}\right)}, i=1, \cdots, N$.

Normalized weights. $\omega_{k}^{i}=\frac{\omega_{k}^{i}}{\sum_{i=1}^{N} \omega_{k}^{i}}$.

Step 5. Determine whether resampling is needed. Calculate the effective sampling scale of particles $M_{\text {eff, }}, N_{\text {eff }}=\frac{1}{n}$. If $M_{\text {eff }}<N$, resampling is needed, otherwise skip to

Step 7.

$$
\sum_{i=1}^{n}\left(\omega_{k}^{i}\right)^{2}
$$

Step 6. Resampling. The total number of particles is unchanged and resampling is based on the particle weight, that is, large particles are copied, whereas small particles are abandoned. Thus, the number of copies of each particle is directly proportional to the particle weight. After completing particle update, the weight of all particles will be set to $\omega^{i}{ }_{k}=1 / N$.

Step 7. Estimate target location. State particles with weights of $x_{k}$ can be written as

$$
p\left(x_{k}\right)=\left\{x_{k}^{i}, \omega_{k}^{i}\right\}_{i=1}^{N} \text {. }
$$


The update state estimate is described as $\hat{x}_{k}=\sum_{i=1}^{N} \omega_{k}^{i} x_{k}^{i}$.

Step 8. Make $k=k+1$ and repeat iteration steps $2-8$ for complete target tracking.

\section{Experimental Results and Discussion}

\subsection{Simulation parameters}

In order to more realistically simulate the motion trajectory of the target, consider the motion state of the moving target in three directions. In this study, the target is set to move in accordance with the plane variable speed turning model (PVSTM). The simulation parameters used are the attenuation coefficient of 0.03 , the angular velocity of 0.1 , the initial position of the moving target of $(0,0,0) \mathrm{m}$, the velocity component of the moving target in the $\mathrm{X}-, \mathrm{Y}_{-}$, and $Z$-directions of $(4,-4,4) \mathrm{m} / \mathrm{s}$, and the acceleration component of $(0.5,-0.5,-0.5) \mathrm{m} / \mathrm{s}^{2}$. Process noise obeys a Gaussian distribution with zero mean, the noise variance is set to $0.01 \mathrm{~m} / \mathrm{s}^{2}$, and the standard deviation of the distance error is $10 \mathrm{~m}$. The simulation observation time is set to $100 \mathrm{~s}$ and the observation time interval is $1 \mathrm{~s}$.

In this study, the three-dimensional dynamic location tracking process in the urban environment is simulated and verified, and the feasibility of seamless positioning based on GPS, Beidou, and DTMB is determined. It can be seen from the seamless positioning equation that, when the coordinate positions of six signal sources are known, the position information of the target can be calculated. In this study, three GPS satellite signals, two Beidou satellites, and one DTMB signal are used for simulation.

\subsection{Comparison of simulation results between EKF and PF}

In order to verify the effect of the number of particles for PF on the position tracking accuracy, the position tracking effect is verified for PF and EKF. The same simulation model has been selected for comparative analysis in this study, and 100 and 500 particles have been used for simulation. The position tracking trajectory and error are shown in Fig. 1.

Figure 1(a) shows that the target moves in accordance with the PVSTM motion in the threedimensional plane, the tracking trajectory of the PF algorithm is closer to the real trajectory, and the change in trajectory is gentle, which substantially coincides with the real trajectory. As in the PF algorithm, the effect of the tracking trajectory of 500 particles is more distinct than that of 100 particles. However, the EKF algorithm can preferably locate the tracking trajectory of the target, but the deviation between the tracking and real trajectories is larger, and the shaking of the trajectory is somewhat severe. Therefore, it can be seen from the change in tracking trajectory that both PF and EKF can complete the positioning and tracking, but PF shows obvious advantages for the instantaneous precision position, while the instantaneous position of EKF fluctuates and the error changes greatly, which give rise to uncertainty in the real-time positioning of the target motion user. Therefore, we can infer that the positioning tracking accuracy of the PF algorithm is higher than that of the EKF algorithm. 


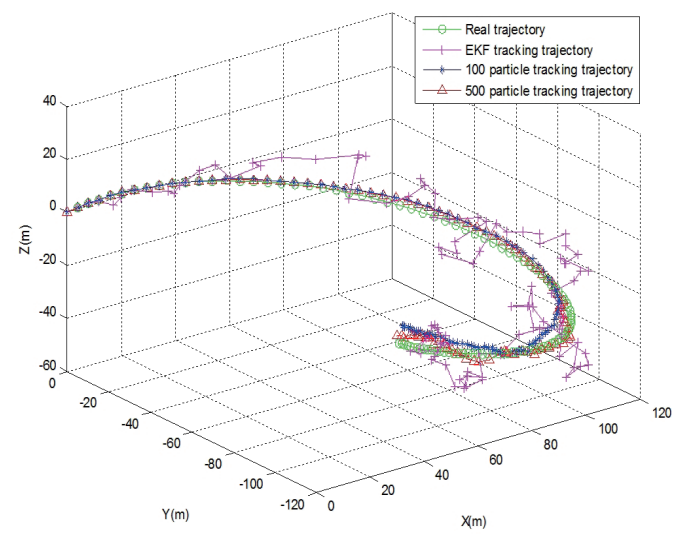

(a)

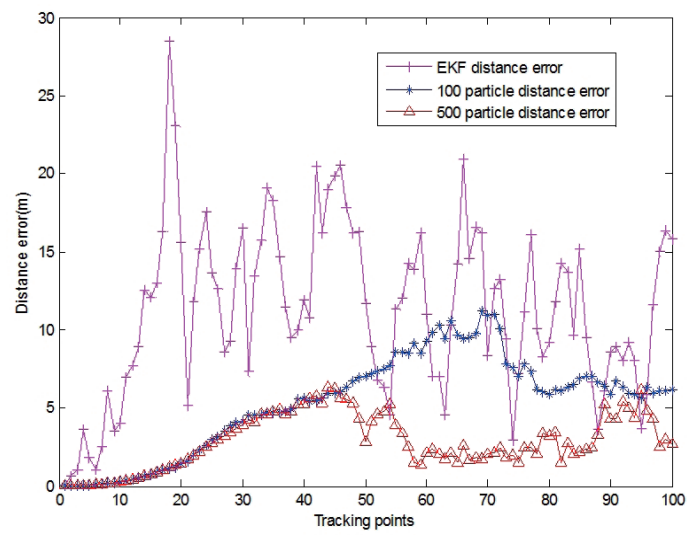

(b)

Fig. 1. (Color online) Results of EKF and PF: (a) tracking trajectories and (b) tracking errors.

Figure 1(b) shows that the tracking accuracy of 500 particles in the PF algorithm is higher than that of 100 particles, and that the tracking accuracy of the PF algorithm is obviously superior to that of the EKF algorithm. We can see that the tracking error of 500 particles in the $\mathrm{PF}$ algorithm is significantly less than that of 100 particles since the moment of 45 observation points; the error of 500 particles ranges from 0 to $5 \mathrm{~m}$, and the error of 100 particles ranges from 5 to $11 \mathrm{~m}$. Moreover, the tracking error fluctuation change of EKF ranges from 0 to $30 \mathrm{~m}$, the maximum tracking distance error is about $28 \mathrm{~m}$, and the minimum tracking distance error is about $1 \mathrm{~m}$.

\subsection{Tracking trajectory and error in three directions}

For further comparative analysis for the PF and EKF algorithms, the positioning tracking trajectories and distance errors of the two filtering algorithms are displayed in Fig. 2 in three directions. From the position tracking simulation results in Fig. 2, we can see that the distance tracking error fluctuation of EKF is volatile when the target movement shifts; thus, EKF is sensitive to the direction change of the moving target, but PF is not, and all the tracking trajectories of $\mathrm{PF}$ are very gentle in all observation points. In addition, when the target movement shifts, the trajectory of 500 particles is closer to the true trajectory than that of 100 particles. Thus, the PF algorithm is more suitable for seamless positioning systems than the EKF algorithm, has a gentler filtering process, and gives more reliable filtering results.

\subsection{Statistical analysis results of EKF and PF}

Statistical analysis results are shown in Table 2, the error mean of 500 particles is $2.8736 \mathrm{~m}$, the error mean of 100 particles is $5.3601 \mathrm{~m}$, and the error mean of the EKF algorithm is $11.2065 \mathrm{~m}$. Comparative analysis shows that the error mean of 500 particles is minimum, the error mean of 100 particles is greater than that of 500 particles, and the mean error of EKF is maximum. Similarly, the standard deviation and variance follow similar laws. Therefore, the higher the 


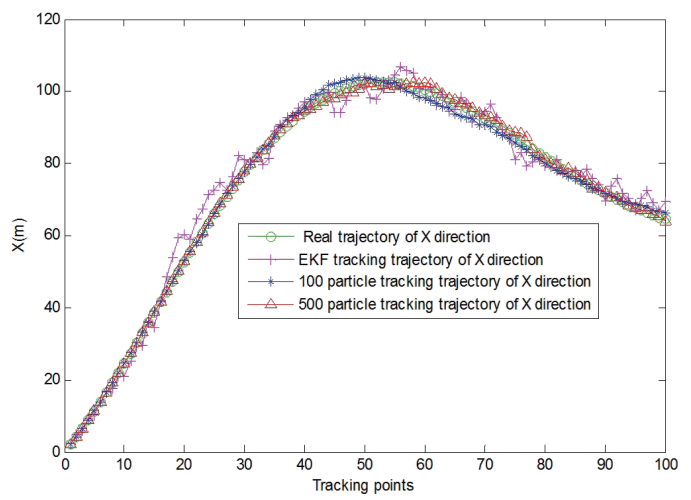

(a)

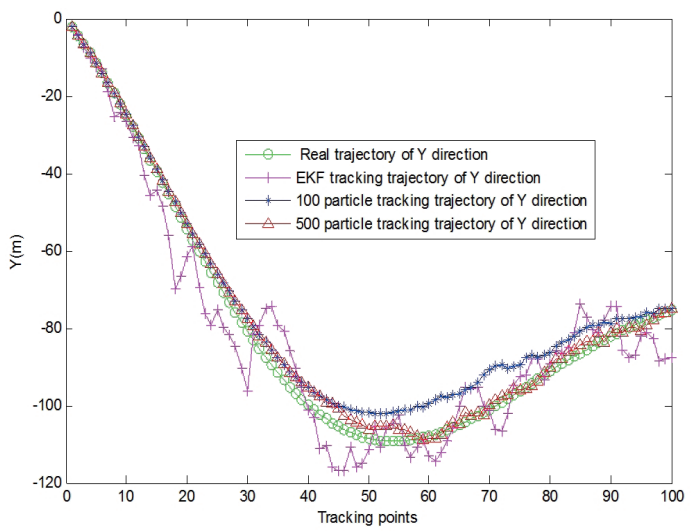

(c)

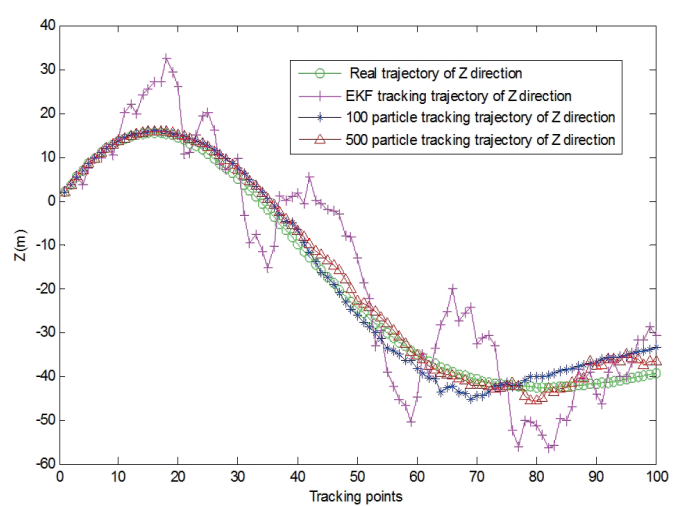

(e)

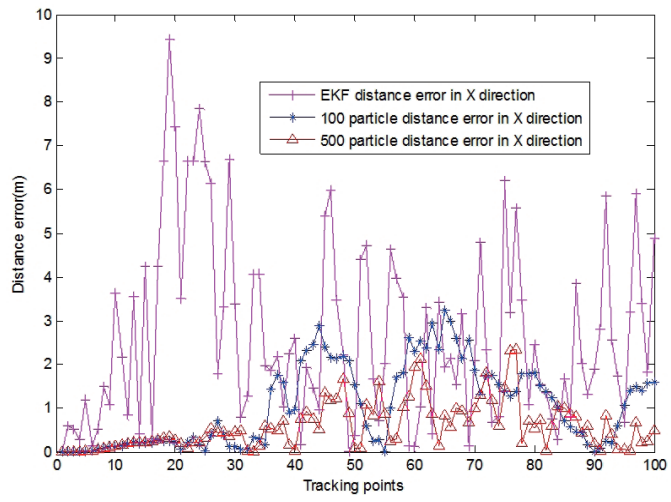

(b)

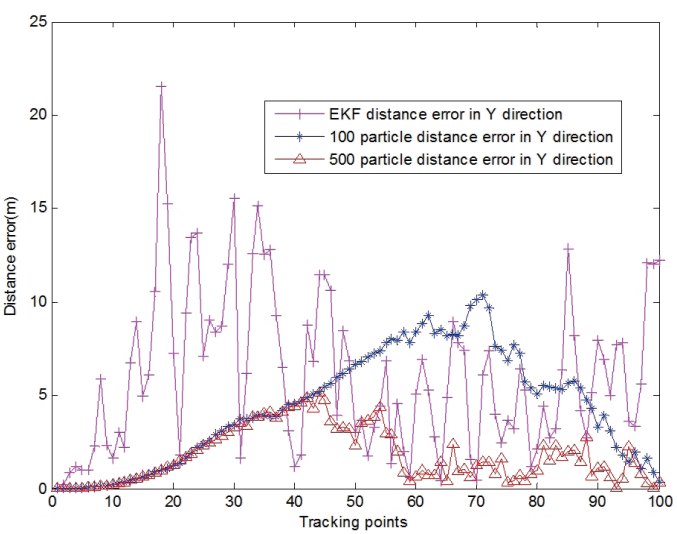

(d)

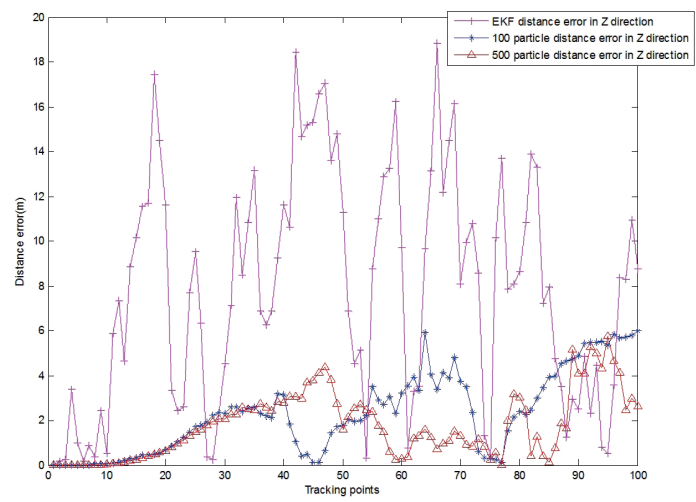

(f)

Fig. 2. (Color online) Simulation results in three directions: (a) tracking trajectory in $X$-direction, (b) tracking error in $X$-direction, (c) tracking trajectory in $Y$-direction, (d) tracking error in $Y$-direction, (e) tracking trajectory in $Z$-direction, and (f) tracking error in Z-direction.

Table 2

Statistical analysis results of EKF and PF.

\begin{tabular}{lccc}
\hline Algorithm & Error mean $(\mathrm{m})$ & Standard deviation $(\mathrm{m})$ & Variance \\
\hline EKF & 11.2065 & 5.4361 & 29.5513 \\
PF (100 particles) & 5.3601 & 3.1325 & 9.8124 \\
PF (500 particles) & 2.8736 & 1.7603 & 3.0988 \\
\hline
\end{tabular}


number of particles used for tracking and positioning, the higher the positioning tracking accuracy, that is, the error mean, standard deviation, and variance are all small, and the tracking precision of the PF algorithm is superior to that of the EKF algorithm.

\section{Conclusions}

In this study, because the characteristics of a single positioning system cannot meet the demand of seamless positioning, a novel seamless navigation system based on GPS, Beidou, and DTMB was proposed. Simultaneously, seamless positioning technology solutions were provided. Also, the observation and state equations of the combination system were presented. Because of the nonlinear characteristics of the combination equation, target tracking was successfully completed by using the nonlinear filtering algorithm under PVTM, and the location tracking effects of the PF and EKF algorithms were comparatively analyzed.

\section{Acknowledgments}

This work was supported financially by the National Natural Science Foundation of China (41861054 and 41561083) and the Yunnan Province Science and Technology Department and Education Department Project (2015FA016, 2017FH001-067, 2017FH001-117, and 2016ZDX127), China.

\section{References}

1 J. X. Yang, X. B. Wang, M. J. Rahman, S. I. Park, H. M. Kim, and Y. Y. Wu: IEEE Trans. Broadcast. 58 (2012) 347. https://doi.org/10.1109/TBC.2012.2191693

2 G. B. Moon, G. I. Jee, and J. G. Lee: Int. J. Control Autom. Sys. 9 (2011) 574. https://doi.org/10.1007/s12555011-0318-9

3 L. L. Dai, Z. C. Wang, J. Wang, and Z. X. Yang: IEEE Trans. Consum. Electron. 56 (2010) 374. https://doi. org/10.1109/TCE.2010.5505942

4 M. Rabinowitz, and J. J. Spilker: IEEE Trans. Broadcast. 51 (2005) 51. https://doi.org/10.1109/ TBC.2004.837876

5 L. Chen, H. E. Feng, M. Fend, and L. N. Wu: Signal Process. 25 (2009) 674.

6 J. Wang, X. Y. Ji, Y. Zhang, L. L. Dai, and K. L. Hu: J. Tsinghua Uni. Sci. Tech. 51 (2011) 729.

7 P. B. S. Harsha, and D. V. Ratnam: IEEE Geosci. Remote Sens. Lett. 13 (2016) 1280. https://doi.org/10.1109/ LGRS.2016.2581207

8 J. M. Pak, C. K. Ahn, S. Peng, and M. T. Lim: Neurocomputing 173 (2016) 645. https://doi.org/10.1016/ j.neucom.2015.08.011

9 R. K. Jaiswal, and C. D. Jaidhar: Wireless Netw. 23 (2017) 2021. https://doi.org/10.1007/s11276-016-1265-4

10 P. Yang and W. Y. Wu: IEEE Trans. Ind. Electron. 61 (2014) 5641. https://doi.org/10.1109/TIE.2014.2301737

11 D. M. Sheinson, J. Niemi, and W. Meiring: Math. Biosci. 255 (2014) 21. https://doi.org/10.1016/j.mbs.2014.06.018

12 M. Boccadoro, G. D. Angelis, and P. Valigi: Wireless Netw. 18 (2012) 579. https://doi.org/10.1007/s11276-012$0420-9$ 


\section{About the Authors}

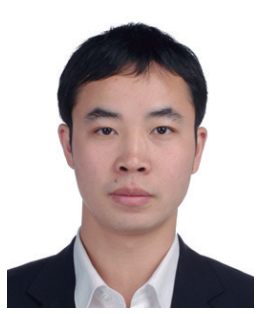

Cheng-Biao Fu is a doctoral graduate student of the Faculty of Land Resource Engineering, Kunming University of Science and Technology. He received his B.S. and M.S. degrees from Chongqing University of Posts and Telecommunications, China, in 2005 and 2009, respectively. Since 2015, he has been a lecturer at Qujing Normal University. His research interests are in information identification and processing, hyperspectral remote sensing and Internet of Things. (fucb@mail.qjnu.edu.cn)

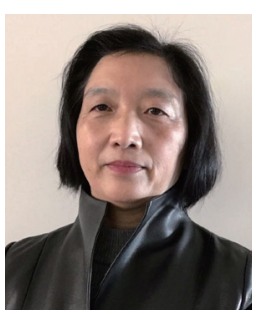

Shu Gan received her B.S. degree from Wuhan University of Surveying and Mapping Science and Tec, China, in 1987. She received her M.S. degree from Southwest Forestry University, China, in 1995, and her Ph.D. degree from Zhejiang University, China, in 2000. Since 2002, she has been a professor at Kunming University of Science and Technology. Her research interests are in spatial data modeling, surveying technology and remote sensing. (sgbf@kmust.edu.cn)

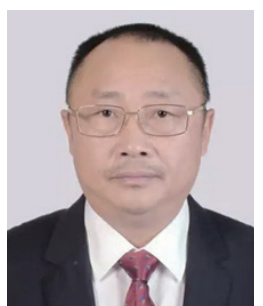

Xi-Ping Yuan received his B.S. degree from Wuhan University of Surveying and Mapping Science and Tec, China, China, in 1987, and he received his Ph.D. degree from Kunming University of Science and Technology, China, in 2007. Since 2003, he has been a professor at Kunming University of Science and Technology University. His research interests are in surveying technology and remote sensing. (YXP@kmust.edu.cn)

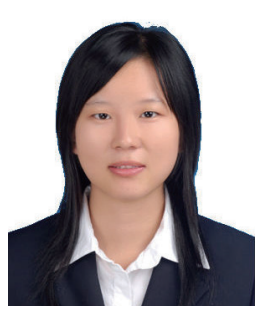

An-Hong Tian is a doctoral graduate student of the Faculty of Land Resource Engineering, Kunming University of Science and Technology. Since 2017, she has been an associate professor at Qujing Normal University. Her research interests are in remote sensing and artificial intelligence.

(tianah@mail.qjnu.edu.cn) 